\title{
Robust AND ADAPTIVE CONTROL OF TWIN Rotor MIMO SYSTEM
}

\author{
Petr Chalupa , Jakub Novák
}

Tomas Bata University in Zlin, Faculty of Applied Informatics, nám. T.G.Masaryka 5555, Zlin 76001, Czech Republic

\begin{abstract}
The paper is focused on control of a Twin Rotor MIMO System - a laboratory model constructed by Feedback Instruments Ltd. The system consists of a two rotors attached to a bar which can freely turn around two axes. System behaviour resembles a simple helicopter. Significant cross-coupling can be observed and the system is nonlinear. A model in the MATLAB/Simulink environment, which was developed within the scope of previous work, was used to design and verify both adaptive and robust controllers of the system. Real-time control using both approaches is presented in the paper. Results of adaptive and robust control are compared using both control courses and integrative criteria.
\end{abstract}

Keywords: Adaptive control; Robust Control; Twin Rotor MIMO System; Modelling; Real-time control
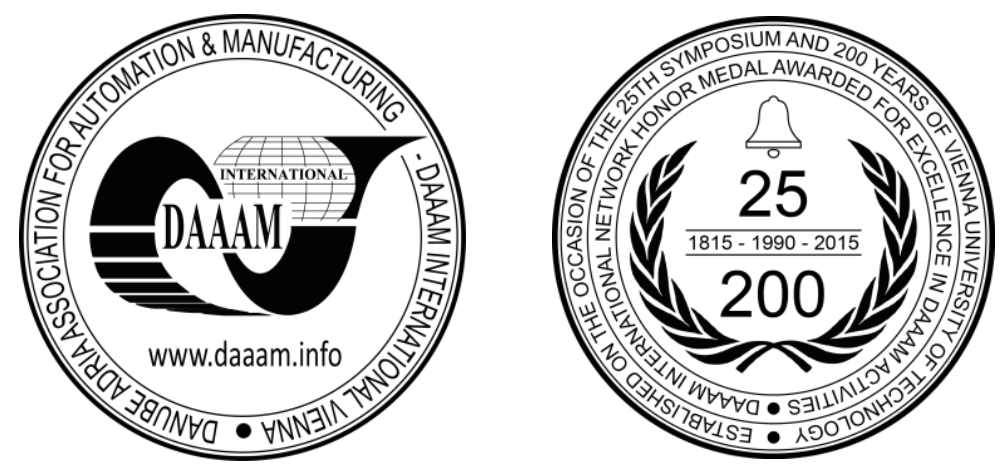

This Publication has to be referred as: Chalupa, P[etr] \& Novak, J[akub] (2016). Robust and adaptive control of twin rotor MIMO system, Proceedings of the 26th DAAAM International Symposium, pp.0133-0141, B. Katalinic (Ed.), Published by DAAAM International, ISBN 978-3-902734-07-5, ISSN 1726-9679, Vienna, Austria DOI:10.2507/26th.daaam.proceedings.019 


\section{Introduction}

Control of oscillatory nonlinear plants is a challenging problem. The laboratory real-time plant "Twin Rotor MIMO System" (TRMS) [1] developed by Feedback Inc. represents such a system with two inputs and two outputs with significant cross-couplings. A nonlinear model of this plant was developed in the scope of previous work in the MATLAB/Simulink environment [2]. The model was derived using combination of black box modelling [3], [4] and the first principles modeling (mathematical-physical analysis of the plant) [5]. This approach is known as grey box modeling [6].

The goal of the work presented in this paper was to use this model for design of a controller for the TRMS. A model, which represents the plant well, can considerably reduce testing time of different control approaches. Then only promising control strategies are applied to the real plant and verified.

The paper is focused on adaptive control design and on robust control design. Self-tuning controllers - a subset of adaptive controllers - are used in the paper. The self-tuning controllers [7] are based on on-line identification of controlled process and the current model is used for computation of parameters of the controller. This approach enables usage of standard control design algorithms based on model of the controlled system. Usually linear model are used and combined with on-line identification of model parameters allow for dealing with non-linear and time-varying processes. The self-tuning controllers presented in the paper are based on ARX models of the system [8].

The other presented control approach - the robust control approach allows obtaining a stabilizing controller not only for a nominal controlled system but also for a wider set of controlled systems [9]. The set can be defined in various ways. This paper deals with parametric uncertainty, namely interval uncertainty [10].

There are many approaches to control a MIMO system [11] but a simple idea of decoupling the MIMO system into a set of Single Input Single Output (SISO) systems in utilized in the paper.

The paper is organized as follows. Section 2 shortly presents the Twin Rotor MIMO System. Adaptive control design is presented in Section 3 while robust control design is presented in Section 4. The real-time experiments and comparison of controllers are described in Section 5.

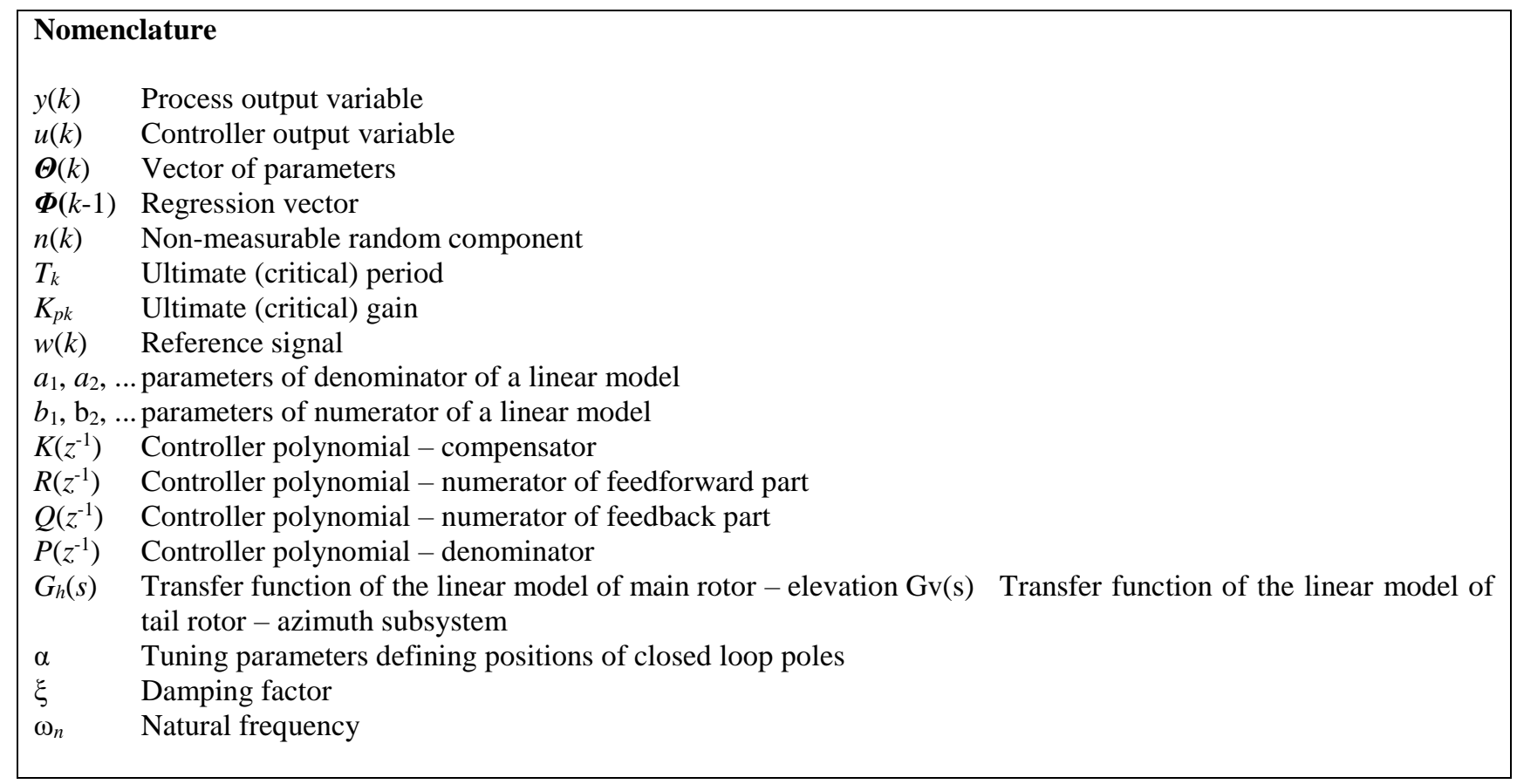

\section{Twin Rotor MIMO System}

A photograph of the Twin Rotor MIMO System is presented in Fig. 1. The system is used to demonstrate the principles of a non-linear multi-input multi-output (MIMO) system with significant cross-coupling. Its behavior resembles a helicopter but contrary to most flying helicopters the angle of attack of the rotors is fixed and the aerodynamic forces are controlled by varying the speeds of the motors. Significant cross-coupling is observed between the actions of the rotors, with each rotor influencing both angle positions [1].

There are two propellers driven by DC-motors at both ends of a beam, which is pivoting on its base. The joint allows the beam to rotate in such a way that its ends move on spherical surfaces. There is a counter-weight fixed to the beam and it determines a stable equilibrium position. The controls of the system are the motors supply voltages. The measured signals are position of the beam in the space, i.e. two position angles [12]. 


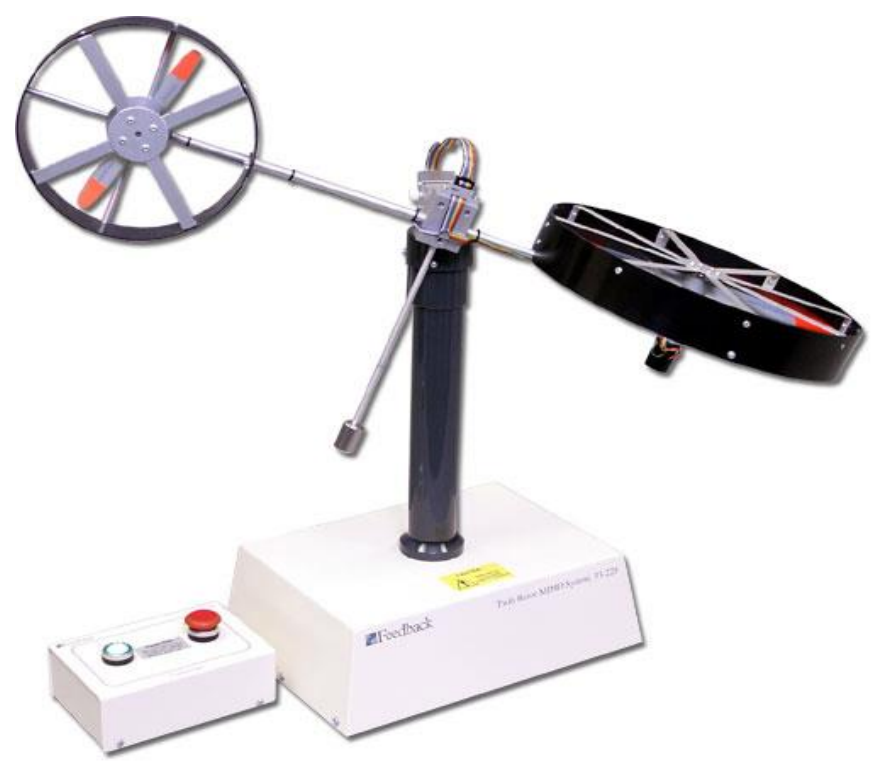

Fig. 1. Twin Rotor MIMO System

\section{Design of adaptive controller}

Self-tuning control, which is a subset of adaptive control, is based on recursive estimation of unknown system characteristics. Using this knowledge, appropriate methods can be employed to design the optimal controller. Selftuning controllers use the combination of the recursive process identification based on a selected model process and a controller synthesis based on knowledge of parameter estimates of controlled process.

Presently, most of the STCs are based on the Certainty Equivalence (CE) Principle. The basic principle of CE is that the model uncertainty is not considered. The parameter estimates of the process model, which are obtained by recursive identification, are used for the controller design. It is assumed that values of these estimates correspond to their actual values.

\subsection{Recursive identification}

The regression (ARX) model of the following form

$$
y(k)=\boldsymbol{\Theta}^{T}(k) \boldsymbol{\Phi}(k-1)+n(k)
$$

is used in the identification part of the designed controller algorithms, where

$$
\boldsymbol{\Theta}^{T}(k)=\left[a_{1}, a_{2}, \ldots, a_{n a}, b_{1}, b_{2}, \cdots, b_{n b}\right]
$$

is the vector of the parameters and

$$
\boldsymbol{\Phi}^{T}(k-1)=[-y(k-1),-y(k-2), \ldots,-y(k-n a), u(k-1), u(k-2), \ldots, u(k-n b)]
$$

is the regression vector. The non-measurable random component $n(k)$ is assumed to have zero mean value $E[n(k)]=0$ and constant covariance (dispersion) $R=E\left[n^{2}(k)\right]$.

The recursive least squares method for calculating of parameter estimates $\hat{\boldsymbol{\Theta}}(k)$ is utilized. Using the pure least squares method, the influence of all input and output data to the parameters estimates is the same. This property can be inconvenient for example when identifying a system with time-varying parameters. In case of Twin Rotor MIMO System, the non-linear plant is modelled by a linear system. The optimal linear model changes with respect to current state of nonlinear real-time pant. In this case, it is better to use least squares method with exponential forgetting where the influence of latter data to the calculation of the parameter estimates is greater than the influence of older data. The exponential forgetting method can be further improved by adaptive directional forgetting [13] which changes forgetting coefficient with respect to changes of input and output signal. 


\subsection{Controller algorithms}

Several controller types were tested and two of them are presented in this paper:

- $\quad$ PID controller with Tahakashi modification of Ziegler-Nichols method [7]

- two-degree-of-freedom (2DOF) controller based on pole placement method [7]

The control law of Takahashi controller is given by the following equations:

$u(k)=K_{p}\left\{-y(k)+y(k-1)+\frac{T_{0}}{T_{I}}[w(k)-y(k)]+\frac{T_{D}}{T_{0}}[-y(k)+2 y(k-1)-y(k-2)]\right\}+u(k-1)$

where

$$
K_{p}=0.6\left(1-\frac{T_{0}}{T_{k}}\right), \quad T_{I}=\frac{K_{p} T_{k}}{1.2 K_{p k}}, \quad T_{D}=\frac{3 K_{p k} T_{k}}{40 K_{p}}
$$

Parameters $T_{k}$ and $K_{p k}$ represent ultimate (critical) period and ultimate (critical) gain. These parameters can be calculated from a linear model.

The control law of the 2DOF controller is calculated according to the following equation:

$u(k)=\frac{1}{K\left(z^{-1}\right)}\left[\frac{R\left(z^{-1}\right)}{P\left(z^{-1}\right)} w(k)-\frac{Q\left(z^{-1}\right)}{P\left(z^{-1}\right)} y(k)\right]$

The scheme of the control loop with 2DOF controller is presented in Fig 2.

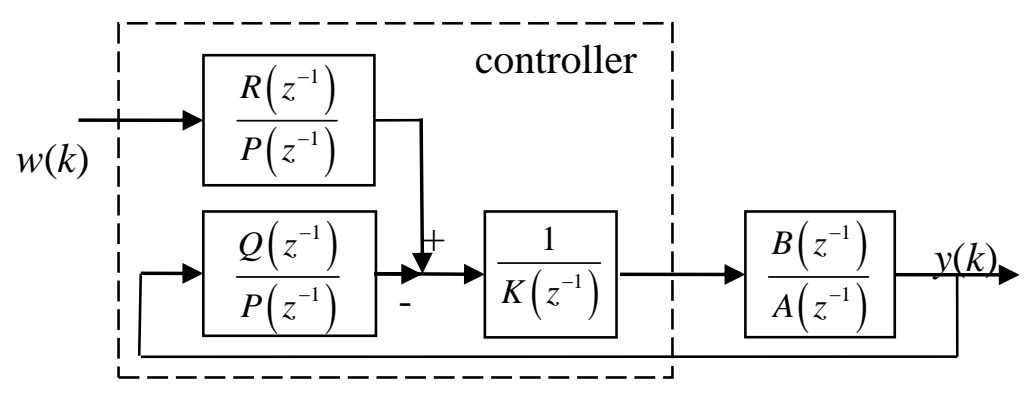

Fig. 2. 2DOF controller

Parameters of controller polynomials $R\left(z^{-1}\right), P\left(z^{-1}\right), Q\left(\mathrm{z}^{-1}\right)$ and $K\left(\mathrm{z}^{-1}\right)$ can be calculated by pole placement method [7].

\section{Design of robust controller}

A design of robust controllers for the Twin Rotor MIMO System is based on parametric uncertainty approach. The parametric uncertainty can be used for both continuous-time and discrete systems [14].

The controlled plant was analyzed to obtain an interval uncertainty of the linear model of the system. For the control purposes, the Twin Rotor MIMO System was considered as two independent systems

- control voltage of the main rotor (input) - elevation (output)

- control voltage of the tail rotor (input) - azimuth (output)

The cross-couplings are considered as disturbances in this case. Various step changes of the control input were applied to the plant, time responses of plant were measured and corresponding linear models were identified.

\subsection{Identification of main rotor - elevation subsystem}

Step changes of the control voltage of main rotor usually lead an oscillatory response of the elevation output. This system was modeled by a $3^{\text {rd }}$ order linear system: 
$G_{h}(s)=\frac{b_{0 h}}{a_{3 h} s^{3}+a_{2 h} s^{2}+a_{1 h} s+1}$

Identification was performed in least squares sense using MATLAB function fminsearch. Results are summarized in Table 1.

\begin{tabular}{lcc}
\hline Parameter & Minimal value & Maximal value \\
\hline$b_{0 h}$ & 60 & 157 \\
$a_{3 h}$ & 0.23 & 1.30 \\
$a_{2 h}$ & 0.22 & 2.35 \\
$a_{1 h}$ & 0.81 & 2.74 \\
\hline
\end{tabular}

Table 1. Intervals of the main rotor - elevation model

Common notation for writing transfer functions of systems with interval uncertainties uses square brackets:

$$
G_{h}(s)=\frac{[60 ; 157]}{[0.23 ; 1.30] s^{3}+[0.22 ; 2.35] s^{2}+[0.81 ; 2.74] s+1}
$$

Model behavior especially its stability is defined by position of transfer function poles - i.e. roots of the denominator. The interval model was obtained from 29 linear models which were identified from step responses. All models are stable (real parts are smaller than zero) and oscillatory because each model has a pair of complex conjugated poles.

Intervals of denominator of the interval model (8) form a box in the 3D space. Contrary to experiments not all transfer functions formed from the parameters inside this box are stable. The situation is presented in Fig. 3.

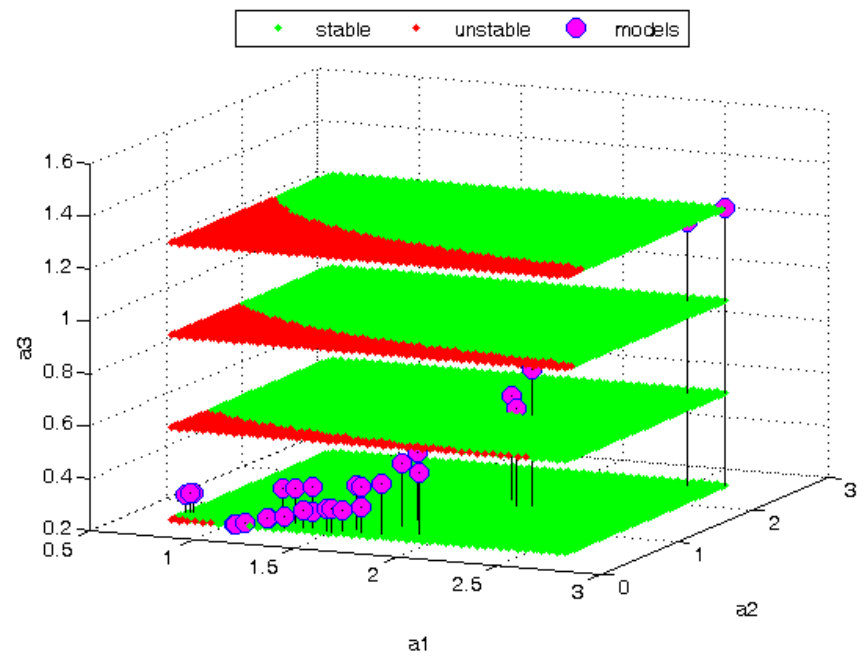

Fig. 3. Stability of interval model of the main rotor - elevation subsystem

Four crosscuts of the box are presented. The crosscuts are parallel to $a_{1 h} \times a_{2 h}$ plane. The upper and the lower crosscut correspond to the maximal and the minimal value of the $\mathrm{a}_{3 h}$ parameter respectively. The other two crosscuts correspond to one third and two thirds between the minimum and maximum of $a_{3 h}$. The red areas correspond to unstable systems while green areas correspond to stable systems. The magenta circles correspond to models identified from step responses.

\subsection{Identification of tail rotor - azimuth subsystem}

The tail rotor - azimuth subsystem was identified in the similar way as the main rotor - elevation subsystem. The main difference consists in fact that 2 nd order models were used, because their accuracy was good enough.

$G_{v}(s)=\frac{b_{0 v}}{a_{2 v} s^{2}+a_{v t} s+1}$ 
The step responses where the plant reached a backstop were omitted from the identification. All the models are stable and most of them are oscillatory. Limits of the model parameters are summarized in Table 2.

\begin{tabular}{lcc}
\hline Parameter & Minimal value & Maximal value \\
\hline$b_{0 v}$ & 75 & 716 \\
$a_{2 v}$ & 2.63 & 6.04 \\
$a_{1 v}$ & 2.49 & 4.55 \\
\hline
\end{tabular}

Table 2. Intervals of the tail rotor - azimuth model

Resulting interval model can be written in the following form:

$G_{v}(s)=\frac{[75 ; 716]}{[2.63 ; 6.04] s^{2}+[2.49 ; 4.55] s+1}$

Intervals of the denominator define a rectangle in $a_{1 v} \mathrm{x} a_{2 v}$ plane. As the denominator is a $2^{\text {nd }}$ order degree polynomial and both $a_{1 v}, a_{2 v}$ are always positive roots of denominator have always negative real part and therefore all the models are stable. Some of the models are oscillatory while the others are aperiodic. The situation is presented in Fig. 4 . The magenta circles correspond to models identified from step responses.

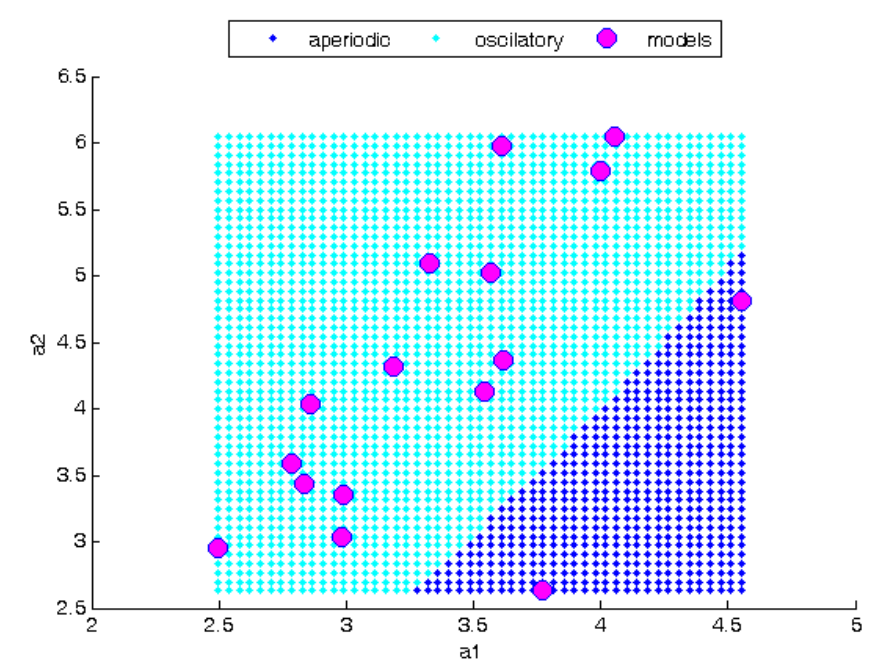

Fig. 4. Behavior of interval model of the tail rotor - azimuth subsystem

\subsection{Design of robust 2 DOF controller}

Several controller types were tested and results of the 2DOF (Two Degree Of Freedom) controllers are presented in this paper. The scheme of the control loop with 2DOF controller is presented in Fig 2. Pole-placement method was used to calculate controllers' polynomials $R(s), P(s), Q(\mathrm{~s})$ and $K(\mathrm{~s})$. Detail can be found for example in [7].

Pole placement method is based on fixing poles of closed loop to the desired positions. For robust control of a system with interval uncertainties the controller is required to guarantee stability of closed loop for any combination of parameters from the given intervals. Unfortunately coefficients of the characteristic polynomial of the closed loop are not independent. Therefore Kharitonov polynomials cannot be used for stability testing [15].

Solving the robust closed loop stability is theoretically complicated task. Hence simplified approach was used:

1. Controller polynomials were calculated with respect to given position of poles and a nominal system.

2. Coefficient values equally spread through an interval were generated for each uncertainty interval.

3. Stability of the closed loop was tested for each combination of coefficient values.

Coefficients of the nominal system were defined as midpoints of the uncertainty intervals. Five coefficient values were generated for each interval. It leads to $5^{4}=625$ combinations (systems) for the main rotor - elevation subsystem and $5^{3}=125$ combinations (systems) for the tail rotor - azimuth subsystem.

Pole positions were defined in the simplest possible way: one multiple real pole was used. Hence the characteristic polynomial of the closed loop has the following form: 
$D(s)=(s+\alpha)^{n}$

Whole task of computing the robust controller can be seen as an optimization problem with the goal of stabilizing all the generated systems and the pole position as a tuning parameter. This approach does not guarantee stability of all possible coefficient combinations but its results in real-time environment were good - no unstable behavior of the closed loop was observed.

\section{Real-Time experiments}

\subsection{Self-tuning controllers}

This section presents several real-time experiments from a huge set of experiments performed. A sampling period of $T_{0}=0.1 \mathrm{~s}$ was used for all experiments. The elevation control courses of Takahashi controller are presented in Fig 5.

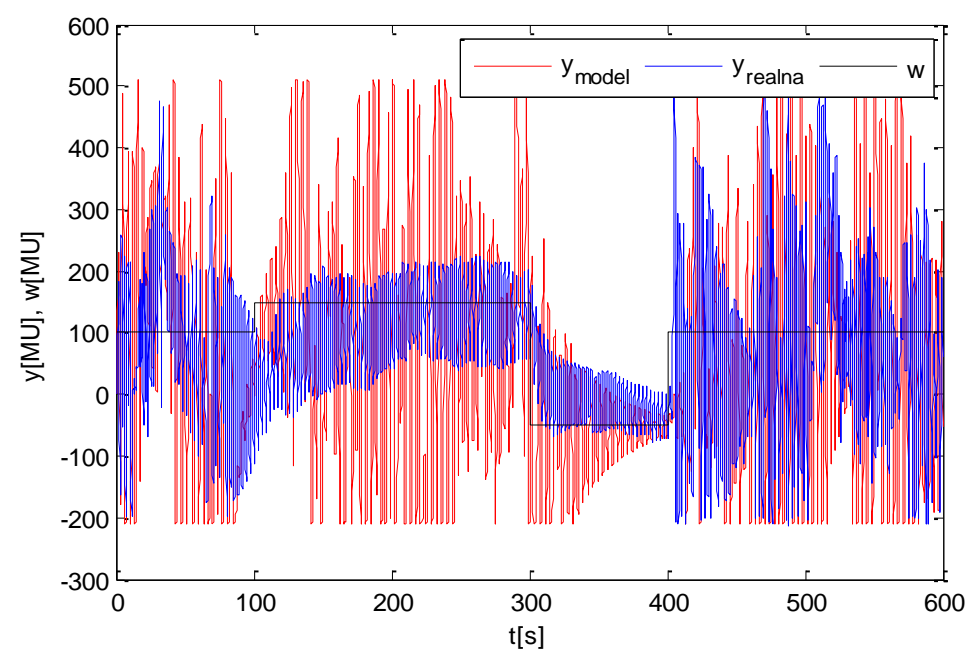

Fig. 5. Elevation control course using Takahashi controller

It can be observed that performance of Takahashi controller is not satisfactory. The course of the output in case of the nonlinear model is even worse comparing to control course of the real plant. Control course of elevation and azimuth using 2DOF controller is presented in Fig. 6.
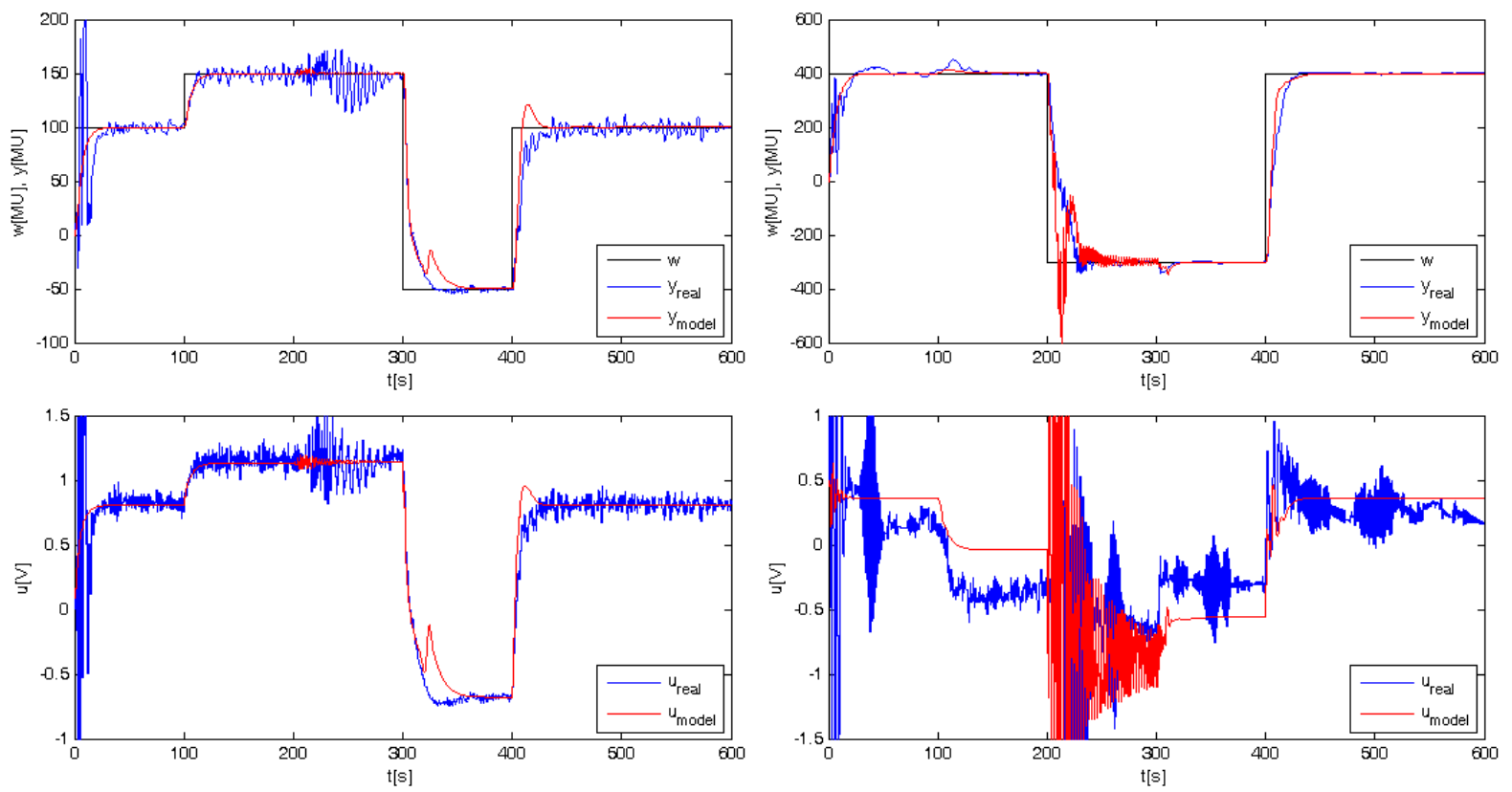

Fig. 6. Elevation (left) and azimuth (right) control course using 2DOF adaptive controller 
The poles of the closed loop were set to obtain behavior of the closed loop similar to the $2^{\text {nd }}$ order system with characteristic polynomial

$$
s^{2}+2 \xi \omega_{n} s+\omega_{n}^{2}
$$

where damping factor was set to $\xi=1.5$ and natural frequency $\omega_{n}=0.4$. The performance of this controller was acceptable for both elevation and azimuth control.

\subsection{Robust control}

This section presents several real-time experiments from a huge set of experiments performed. A sampling period of $T_{0}=0.01 \mathrm{~s}$ was used for sensors and actuators in all experiments. Optimal value of pole position for elevation control was $\alpha_{v}=1.7$ (i.e. multiple pole in position -1.7) and optimal value for azimuth control was $\alpha_{h}=0.6$ (i.e. multiple pole in position -0.6). Elevation control and azimuth control were performed simultaneously to verify effect of cross-couplings. Courses of elevation and azimuth control are presented in Fig. 7. Control of both real-time plant and nonlinear model derived in Section 4 are presented.
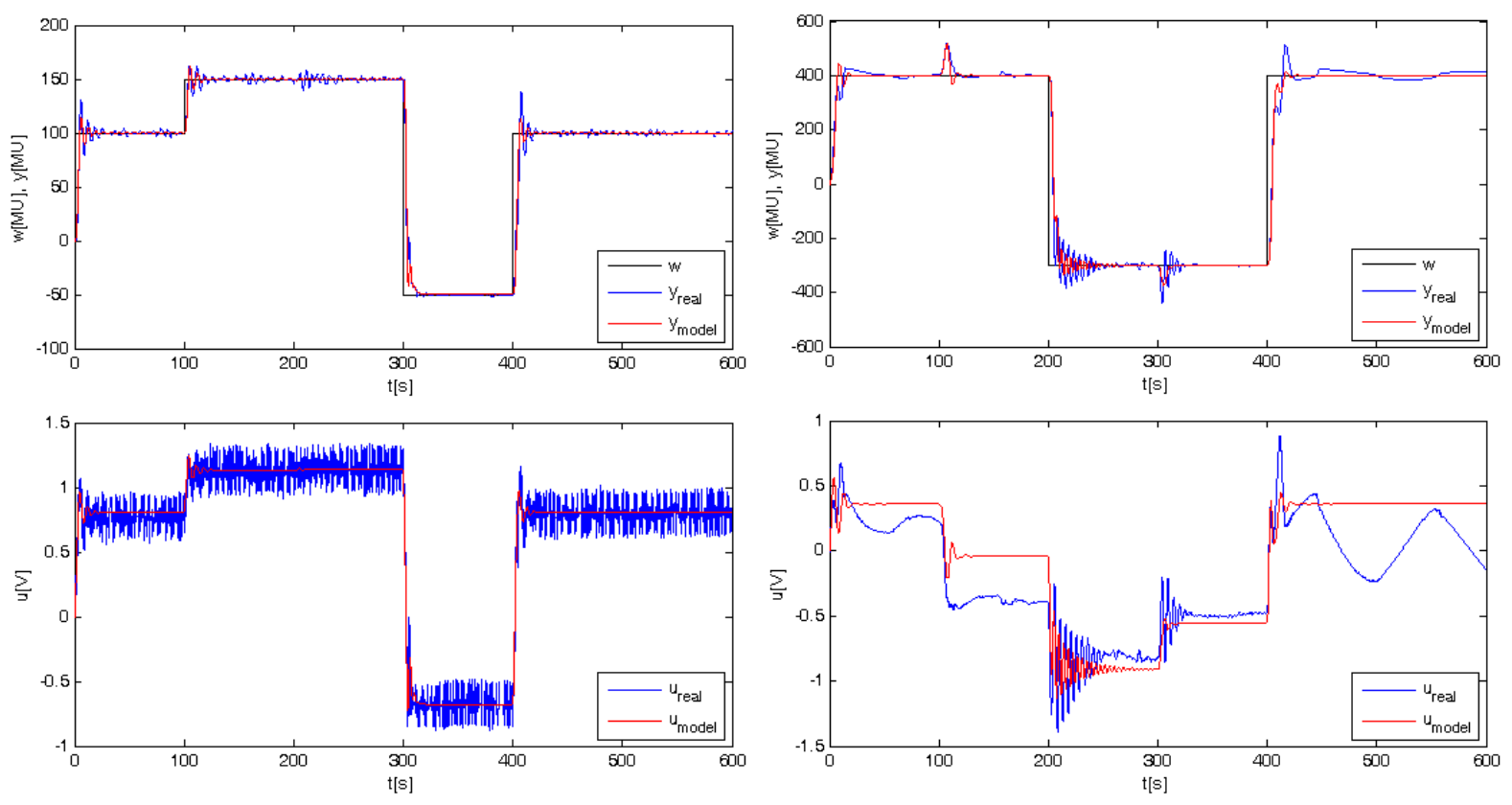

Fig. 7. Elevation (left) and azimuth (right) control using robust controller

A satisfactory control behavior with a good reference tracking was observed. A difference between nonlinear model and real-time plant was observed for control signal of the tail rotor.

\section{Conclusion}

Self-tuning control deign based on linearized model of the plant was designed for control of the Twin Rotor MIMO System. Control courses of the real plant and of the nonlinear Simulink model were compared. Well known Takahashi controller is not suitable for the given plant. On the other hand, performance of a simple 2DOF controller was significantly better. A simple robust control technique was presented and successfully verified in real-time conditions. Further improvement can be acquired by implementing better pole placement. Usage of several different poles would lead to even better control courses than the courses presented in the paper.

Quantitative comparison of control courses presented in Fig. 6 and Fig. 7 is performed in Table 3

\begin{tabular}{lcccc}
\hline \multirow{2}{*}{ Controller } & $\sum e^{2}$ & $\sum \Delta u^{2}$ & $\sum e^{2}$ & $\sum \Delta u^{2}$ \\
\hline Adaptive 2DOF & 698 & 0.0193 & 11630 & 0.0468 \\
Robust & 332 & 0.0070 & 7590 & $2,59 \mathrm{e}-5$ \\
\hline
\end{tabular}

Table 3. Comparison of control courses 
Presented control design approaches can be generally used for a much larger set of controlled systems. On the other hand, as the stability was not studied in the paper from the theoretical point of view, it should be checked very carefully when applying presented approaches in real-time conditions.

Further work will be focused on obtaining even better performance of the controllers. Adaptive controllers based on LQ approach will be tested an using multiple poles will be used for robust controller.

\section{Acknowledgements}

This work was supported by the Ministry of Education, Youth and Sports of the Czech Republic within the National Sustainability Programme project No. LO1303 (MSMT-7778/2014).

\section{References}

[1] P. Chalupa, J. Přikryl, J. Novák, Modelling of Twin Rotor MIMO System, Procedia Engineering 100 (2015), pp. 249-258.

[2] Feedback Instruments, Twin Rotor MIMO System 33-007-PCI, Crowborough, 2013.

[3] G. P. Liu, Nonlinear identification and control - A neural network Approach. Springer-Verlag, London, 2001.

[4] L. Ljung, System identification: theory for the user. Upper Saddle River, Prentice Hall PTR, N.J., 1999.

[5] D. M. Himmelblau and J. B. Riggs, Basic principles and calculations in chemical engineering. Prentice Hall, N.J., 2004.

[6] K.C. Tan, Y. Li, Grey-box model identification via evolutionary computing, Control Engineering Practice10 (2002), pp. 673-684.

[7] V. Bobál, J. Böhm, J. Fessl, J. Macháček. Digital Self-tuning Controllers: Algorithms, Implementation and Applications. Springer-Verlag, London, 2005.

[8] P. Chalupa, J. Prikryl, J. Novak, Adaptive control of Twin ROTOR MIMO system, in: M. Fikar and M. Kvasnica (Eds.) Proceedings of the 20th International Conference on Process Control (PC), 2015, pp. 314-319.

[9] M. Morari and E. Zafiriou. Robust process control. Englewood Cliffs, Prentice Hall, N.J, 1989.

[10] R. S. Sánchez-Peña and M. Sznaier. Robust systems theory and applications, Wiley, New York, 1998.

[11] Volkova, N. and Prokop, R. Matrix Equation Approach For Mimo Control Design, in: B. Katalinic (Ed.) Annals of DAAAM for 2011 \& Proceedings of the 22nd International DAAAM Symposium, Volume 22, No. 1, Vienna, Austria, 2011, pp. 0225-0226.

[12] Feedback, Twin Rotor Mimo System - Advanced Teaching Manual 1 - 33-007-4M5.

[13] R. Kulhavý, Restricted Exponential Forgetting in Real Time Identification, Automatica 23 (1987), pp. 586-600.

[14] R. Matušů,"Robust Stability Analysis of Discrete-Time Systems with Parametric Uncertainty: A Graphical Approach, International Journal of Mathematical Models and Methods In Applied Sciences 8 (2014), pp. 95-102.

[15] R. Matušů, R. Prokop, Control of Interval Systems Using 2DOF Configuration, Procedia Engineering 100 (2015), pp. $340-344$. 